\title{
Intubação submentoniana na cirurgia bucomaxilofacial: relato de casos clínicos
}

\author{
Submental intubation in maxillofacial surgery: clinical case \\ Intubación submentoniana en cirugía maxilofacial: reporte de caso \\ Breno dos Reis FERNANDES ${ }^{\mathbf{1}}$ \\ Darah Lígia MARCHIORI ${ }^{2}$ \\ Oswaldo BELLOTI NETO ${ }^{3}$ \\ Patrese Pereira de BELLA ${ }^{3}$ \\ Gabriel MULINARI-SANTOS ${ }^{1}$ \\ Manuella Toniazzo TONIAL ${ }^{3}$ \\ Julia Rafaelli FREO ${ }^{3}$ \\ Gabriele Romansin GHELLER ${ }^{3}$ \\ ${ }^{1}$ Faculdade de Odontologia de Araçatuba, UNESP-Univ. Estadual Paulista, 16015-050 Araçatuba-SP, Brasil \\ ${ }^{2}$ Universidade Alto Vale do Rio do Peixe, 89500-000 Caçador-SC, Brasil \\ ${ }^{3}$ Hospital das Clínicas de Teresópolis, 25976-016 Teresópolis-RJ, Brasil
}

\section{Resumo}

A manutenção das vias aéreas em pacientes com lesões maxilofaciais complexas é um desafio para os cirurgiões e anestesiologistas. A intubação submentoniana é uma técnica útil que é menos invasiva que a traqueostomia na proteção das vias aéreas, onde a intubação orotraqueal e nasotraqueal são contraindicadas. Este procedimento evita o uso de traqueostomia e suas complicações, visto que a intubação submentoniana demonstra baixo indice de complicações. Um tubo endotraqueal reforçado flexível e resistente a dobras com conector universal destacável é comumente usado para intubação submental. São apresentados dois casos clínicos utilizando a intubação submentoniana em pacientes com lesões maxilofaciais complexas.

Descritores: Intubação; Ferimentos e Lesões; Cirurgia Bucal.

\section{Abstract}

Maintaining the airways in patients with complex maxillofacial injuries is a challenge for surgeons and anesthesiologists. Submental intubation is a useful technique that is less invasive than tracheostomy in protecting the airways, where orotracheal and nasotracheal intubation are contraindicated. This procedure avoids the use of tracheostomy and its complications, since submental intubation shows a low rate of complications. A flexible, bend-resistant reinforced endotracheal tube with a detachable universal connector is commonly used for submental intubation. Two clinical cases are presented using submental intubation in patients with complex maxillofacial injuries.

Descriptors: Intubation; Wounds and Injuries; Surgery, Oral.

\section{Resumen}

Mantener las vías respiratorias en pacientes con lesiones maxilofaciales complejas es un desafío para los cirujanos y anestesiólogos. La intubación submental es una técnica útil que es menos invasiva que la traqueotomía para proteger las vías respiratorias, donde la intubación orotraqueal y nasotraqueal están contraindicadas. Este procedimiento evita el uso de traqueotomía y sus complicaciones, ya que la intubación submentoniana muestra una baja tasa de complicaciones. Un tubo endotraqueal reforzado flexible y resistente a la flexión con un conector universal desmontable se usa comúnmente para la intubación submentoniana. Se presentan dos casos clínicos con intubación submentoniana en pacientes con lesiones maxilofaciales complejas.

Descriptores: Intubación; Heridas y Traumatismos; Cirugía Bucal.

\section{INTRODUÇÃO}

Fraturas complexas envolvendo o terço superior, médio e inferior da face são denominadas de fratura panfacial e usualmente requerem um plano de tratamento individualizado para cada segmento fraturado ${ }^{1}$.

Pacientes com fraturas complexas do complexo maxilofacial usualmente necessitam de anestesia geral para redução e fixação dessas fraturas $^{2}$. E a manutenção da via aérea, tem grande importância durante a cirurgia. Entretanto, em muitos casos, cirurgião e anestesista competem pelo mesmo espaço durante a cirurgia ${ }^{3}$.

A intubação orotraqueal é um método seguro e preferido para manutenção das vias aéreas. Porém quando existe necessidade de reconstrução do terço inferior da face, a intubação orotraqueal pode interferir na abordagem às fraturas e no bloqueio maxilomandibular ${ }^{4}$.

As alternativas mais comuns para a intubação orotraqueal no trauma maxilofacial é a intubação nasotraqueal, redução das fraturas em etapas separadas e a traqueostomia. Em certos pacientes, entretanto, a intubação nasotraqueal é contraindicada devido a fraturas no terço médio da face, tais como fratura nasal, fratura naso-orbito-etmoidal e da base de crânio, as quais podem acrescentar um risco de comunicação da fossa nasal com a fossa craniana anterior durante a intubação nasotraqueal ${ }^{3,4}$.

A traqueostomia promove uma via aérea definitiva e permite subsequente reparo das fraturas faciais sem interferências nos acessos ao esqueleto facial. Porém a traqueostomia não é o procedimento de eleição e pode ser considerado excessivo, particularmente para aqueles pacientes estáveis na Escala de Coma de Glasgow e que necessita de manutenção da via aérea somente durante o procedimento cirúrgico ${ }^{1,4}$.

A intubação submentoniana é uma alternativa da intubação endotraqueal descrita inicialmente por Hernandez-Altermir em 1986, como alternativa para traqueostomia na cirurgia maxilofacial, promovendo uma via aérea segura e sem interferências. Além dela está associada a um baixo índice de complicações em relação a traqueostomia. ${ }^{2,}{ }^{4}$ Esse trabalho relata 2 casos de fraturas complexas de face, sendo a intubação submentoniana utilizada com sucesso.

\section{CASOS CLÍNICOS}

O presente trabalho descreve os casos clínicos de dois pacientes submetidos a intubação 
submentoniana para redução das fraturas complexas de face no ano de 2018 na Casa de Saúde e Maternidade Santa Mônica, Santo Antônio de Pádua, Rio de Janeiro, Brasil. A técnica e metodologia obedece às seguintes etapas. Na sala cirúrgica, antes de iniciar a anestesia, um tubo endotraqueal aramado é selecionado de acordo com a característica do paciente e o conector do tubo endotraqueal é removido para teste e reinserido ao tubo. Muitos tubos aramados tem o conector colado, dificultando a sua remoção. Remover esse conector facilita a passagem do tubo pela incisão submentoniana. Com a indução da anestesia geral, o paciente é intubado de maneira convencional, uma intubação orotraqueal. Nesse momento, a face e o pescoço do paciente são degermados. Uma incisão em pele de 1.5 a $2 \mathrm{~cm}$ é feita ao longo da borda inferior da mandíbula na linha média ou para-medial (Figura 1). Uma dissecção romba é realizada, atravessando o músculo milohióideo até o soalho da cavidade bucal. O canal transcutâneo criado é alargado com uma pinça Kelly, a qual é passada para a boca e usada para exteriorizar primeiro o balonete do tubo endotraqueal através da incisão. A pinça Kelly é reintroduzida na incisão, nesse momento, o conector do tubo é removido, a abertura do tubo pode ser ocluída para evitar a entrada de saliva e sangue pelo tubo endotraqueal e então ele é exteriorizado pela incisão com auxílio da pina Kelly. O tubo é limpo e o conector é reinserido. $\mathrm{O}$ anestesista verifica se o tubo está em posição adequada através de auscultação e pressão apropriada das vias aéreas. O tubo é então fixado na pele com auxílio de suturas com fio de seda 3-0. Para extubação, o tubo é repassado através da incisão de pele para a cavidade oral e a incisão em pele na região submentoniana é suturada (Figura 2). A extubação pela via submentoniana não é indicada, pois isso aumentaria a inoculação de bactérias da cavidade oral na incisão submentoniana, aumenta a chance de infecção pós-operatória nessa região.

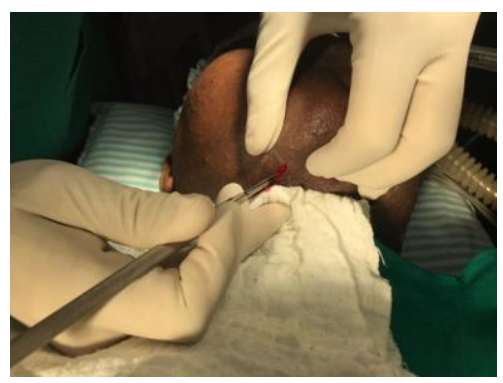

Figura 1: Uma incisão em pele de 1.5 a $2 \mathrm{~cm}$ é feita ao longo da borda inferior da mandíbula na linha média ou para-medial.

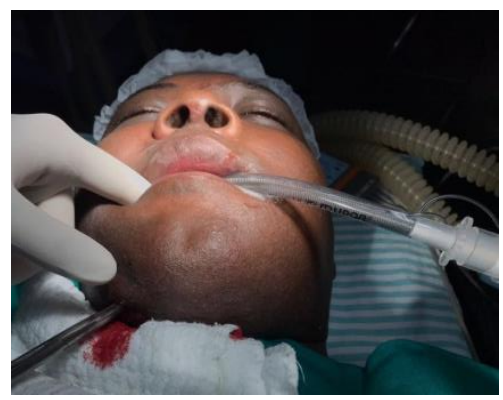

\section{- Paciente 1}

Paciente masculino, 44 anos, melanoderma, vítima de acidente automobilístico, referenciado e atendido na Casa de Saúde e Maternidade Santa Mônica. Apresentava fratura de mandíbula, Le Fort I, zigomática bilateral, orbitária bilateral e nasal. O paciente sem alterações nos exames laboratoriais préoperatórios e risco cirúrgico ASA II, foi trazido ao centro cirúrgico para redução aberta e fixação das fraturas faciais, fratura mandíbular e zigomáticaorbitária via acessos extraorais, Le Fort I via acesso intraoral e com redução aberta da fratura nasal e tamponamento nasal. Realizada anestesia geral, com intubação orotraqueal e o tubo endotraqueal foi reposicionado pela incisão submentoniana (Figura 3).

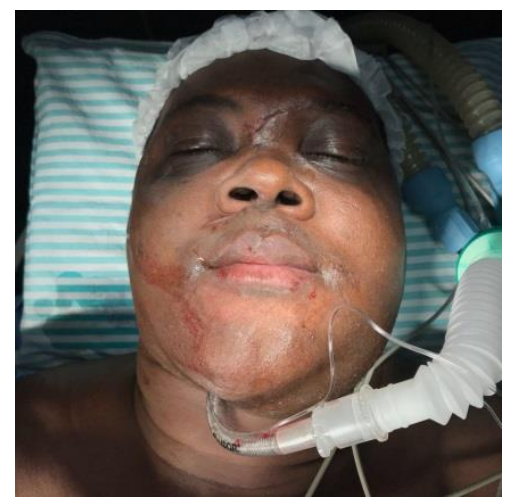

Figura 3: Realizada anestesia geral, com intubação orotraqueal e o tubo endotraqueal foi reposicionado pela incisão submentoniana.

A equipe cirúrgica realizou redução aberta das fraturas e fixação interna com mini-placas e parafusos, com bloqueio maxilomandibular no momento transoperatório. Após o termino do procedimento, o bloqueio maxilomandibular foi removido e o tubo foi relocado para a cavidade oral. A incisão extraoral foi suturada com fio de nylon 4-0 e a incisão intraoral não foi suturada. O paciente foi imediatamente extubado sem complicações.

\section{○ Paciente 2:}

Paciente feminina, 63 anos, leucoderma, vítima de queda de motocicleta, referenciada e atendida na Casa de Saúde e maternidade Santa Mônica. Apresentava fratura de mandíbula, Le Fort I, zigoma esquerdo e nasal. A paciente sem alterações nos exames laboratoriais e risco cirúrgico ASA II, foi trazida ao centro cirúrgico para redução aberta e fixação interna das fraturas faciais. Fratura mandibular e Le Fort I reduzida por acessos intraoral, fratura zigomática esquerda por acesso extraoral e redução fechada da fratura nasal. Realizada indução e intubação orotraqueal e o tubo foi reposicionado pela região submentoniana (Figuras 4 e 5). Foi realizada redução aberta das fraturas e fixação com mini-placas e parafusos, com bloqueio maxilomandibular no momento transoperatório. Após término da cirurgia, o bloqueio maxilomandibular foi removido $\mathrm{e}$ o tubo foi repassado para a cavidade oral. A incisão em pele foi 
suturada com fio de nylon 5-0 e a incisão intraoral não foi suturada. A paciente foi prontamente extubada sem complicações.

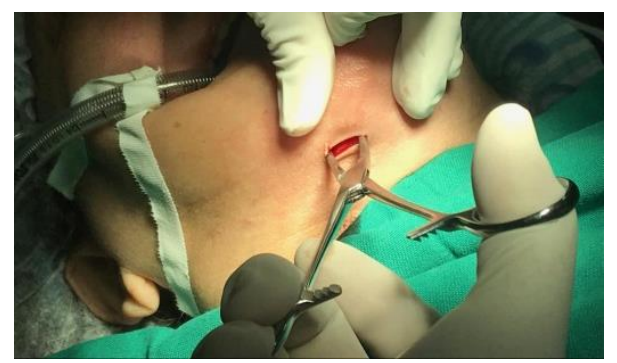

Figura 4: Acesso e divulsão para intubação submentoniana.

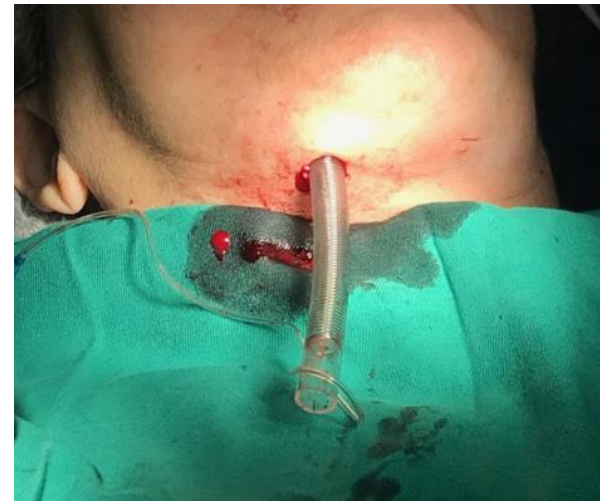

Figura 5: Intubação submentoniana realizada para redução das fraturas faciais.

\section{DISCUSSÃO}

A intubação submentoniana foi introduzida como uma alternativa para a intubação nasal e oral, especialmente em cirurgias maxilofaciais complexas. Ela tem sido utilizada por anos com eficácia e sem relato de complicações importantes ${ }^{5}$. Muitos estudos e autores descrevem a intubação submentoniana como uma técnica rápida, simples e segura para manutenção das vias aéreas no trauma de face ${ }^{5,6}$.

Os critérios de indicação prosposto para a intubação submentoniana são: trauma complexo de crânio e face, necessidade de bloqueio maxilomandibular transoperatório, fratura da pirâmide nasal, presença de patologia nasal, cirurgia ortognática com rinoplastia, ressecção de tumores maxilares, grandes cirurgias da faringe e cirurgia da base do crânio ${ }^{6,7}$.

Apesar de todas as indicações, a intubação submentoniana só deve ser utilizada no transoperatório. Não é indicada para promover uma via aérea prolongada e deve ser convertida para intubação orotraqueal no fim da cirurgia. A traqueostomia está indicada para manutenção da via aérea se ventilação é necessário ${ }^{8}$.

Essa técnica de manejo das vias aéreas também evita lesões das estruturas nasais e o risco de ruptura craniana com inserção do tubo na cavidade craniana, causando danos irreversíveis ao cérebro. ${ }^{5,6}$ A intubação submentoniana não é contraindicada em casos de trauma cervical, já que apenas pode acrescentar uma dificuldade para a técnica cirúrgica e para a intubação ${ }^{6,8}$.
O comprimento da incisão original em pele introduzida por Altemir em 1986 tem 2 centímetros. Entretanto, outros tamanhos da incisão em pele são relatados na literatura, variando de $1,1.5$ a $2 \mathrm{~cm}$. Este comprimento de incisão é o ideal para exteriorizar o tubo flexometálico sem trauma e com o mínimo de tensão ${ }^{6-8}$.

A incisão em pele foi colocada inicialmente no plano paramediano Porém MacInnis e Baig modificaram para uma abordagem no plano medial depois de relatarem algumas complicações. Como dificuldade na passagem do tubo, hemorragia, dano a glândula sublingual e ao ducto da glândula submandibular (Warthon) ${ }^{6,8}$.

A justificativa para a abordagem mediana é evitar grande estruturas como a glândula sublingual, ducto de Warthon e o nervo lingual. Além disso, a possibilidade de hemorragia é reduzida na linha média, pois a rafe milohióidea é avascular ${ }^{7,8}$. No entanto a abordagem para-mediana também é segura, sem riscos as estruturas nobres, que podem ser evitadas com uma dissecação na direção ao plano medial em relação a mandíbula ${ }^{8}$.

A maioria dos cirurgiões utiliza a técnica de exteriorização do tubo com apenas uma pinça hemostática descrita por Altemir em 1986. O uso desta técnica embora eficaz, rápida e conveniente, carrega o risco de um trajeto diferente de saída para o tubo e o balonete. Isso ocorre porque após a exteriorização do balonete, a reinserção da pinça hemostática não pode seguir o mesmo caminho. Podendo o balonete ficar encarcerado nos tecidos e romper no fim do procedimento ${ }^{8,9}$.

Essa complicação levou a muitos cirurgiões elaborar uma alteração da técnica, e sugerir a utilização de duas pinças hemostáticas. Nesta técnica é inserida a primeira pinça hemostática e no momento antes de exteriorizar o balonete, uma segunda hemostática é passada para manter a patencia do túnel e exteriorizar o tubo ${ }^{9}$.

Outra forma de exteriorização é sugerida com a utilização de um espéculo nasal. Após a dissecação romba dos tecidos submentoniano e punção do assoalho da boca, um espéculo é inserido mantendo a patência do túnel, facilitando a passagem do balonete e do tubo sem danos aos tecidos moles e sem entrada de sangue e tecido mole no interior do tubo ${ }^{7,9}$. Métodos minimamente invasivos para realizar o túnel, com utilização de kits dilatadores para traqueostomia também são uma possibilidade. Consistem em ir realizando dissecção romba com os dilatadores até o tamanho suficiente para exteriorizar o tubo. Entretanto, essa técnica não se mostrou superior em relação as demais técnicas ${ }^{10}$.

Uma pequena entrada de sangue na distal do tubo é inevitável nessa técnica, devendo ser limpo com cautela para evitar presença de sangue no interior do tubo. Isso pode ser evitado obstruindo a 
distal do tubo no momento da passagem pelo túnel, com um dedo de luva estéril e removê-lo após a exteriorização ${ }^{8-10}$

A intubação submentoniana é relatada com um baixo índice de complicações, incluindo, extubação acidental, destacamento do balonete, dano ao tubo durante a exteriorização, dano ao cuff do tubo, introdução do tubo no interior do brônquio, obstrução do tubo, infecção na pele, formação de cicatriz, parestesia do nervo lingual, dor, hemorragia, hematoma sublingual, fístula salivar e formação de mucocele. Apesar do número de complicações relatadas, elas raramente ocorrem ${ }^{7,8,10}$.

Geralmente o índice de complicações é baixo, não ultrapassando $10 \%$ no número total dos casos de intubação submentoniana relatados na literatura. Infecção é a complicação mais comum, com uma incidência muito baixa, em torno de $3.5 \%$. Todas as infecções são geralmente superficiais e se resolvem com o uso de soluções antissépticas e antibióticos. E geralmente estão associadas a presença de saliva no interior da ferida, higiene oral deficiente e limpeza inadequada das feridas ${ }^{7,8,11}$.

A presença de cicatriz hipertrófica no pósoperatório geralmente é bem tolerada pelos pacientes. Nenhum tratamento é relatado para cicatriz hipertrófica. A razão para aceitação da cicatriz é o tamanho reduzido, geralmente inferior a $2 \mathrm{~cm} \mathrm{e}$ geralmente são escondidas abaixo da borda mandibular. A tendência para formação de queloides pode ser uma limitação para a utilização da intubação submentoniana ${ }^{8,11}$.

Fístula salivar ou orocutânea ocorre como resultado da comunicação entra a cavidade bucal e pele, com presença de saliva pela fístula. Pode resultar de uma intubação submentoniana prolongada ou uso de técnica imprópria, apresentando-se como uma complicação logo após a extubação ou ainda demorar de 1 a 2 semanas para se manifestar. Se a fistula estiver presente no pós-operatório deve ser realizada sutura por planos e do assoalho da cavidade oral. Porém em certos casos, ela se resolve espontaneamente dentro de 10 a 20 dias $^{7,11}$.

$\mathrm{O}$ deslocamento do tubo, seja parcial ou completa, ocorre como manipulação excessiva do tubo e falta de fixação adequada do tubo. Um aumento da pressão nas vias aéreas e diminuição do dióxido de carbono expirado $\left(\mathrm{ETCO}_{2}\right)$, juntamente com uma queda na saturação de oxigênio $(\mathrm{SpO} 2)$ a menos de $96 \%$ alertará o anestesista da possibilidade de deslocamento do tubo. E geralmente é resolvido pelo anestesista com reposicionamento do tubo. Em vez de deslocamento do tubo para exterior, ele pode ser empurrado inadvertidamente para dentro do brônquio. E geralmente o tubo e deslocado para o brônquio direito, devido a anatomia do mesmo, que é relativamente mais amplo e reto em relação a traqueia em comparação ao brônquio esquerdo. A torção do tubo raramente ocorre, sendo representada por um aumento da pressão nas vias aéreas ${ }^{8,11,12}$.

Durante a exteriorização o balonete pode ser rompido, é uma complicação que pode ser evitada manipulando com cuidado. O melhor método para resolver deve ser a substituição do tubo por um novo tubo endotraqueal ${ }^{8,12}$.

Complicações das glândulas salivares são extremamente raras na intubação submentoniana. As mucoceles e sialoceles podem ser formar pela incorporação de remanescentes da mucosa no interior do túnel orocutâneo. A lesões as glândulas salivares podem ser evitadas com aplicação correta da técnica ${ }^{11}$.

A intubação retromolar é uma técnica segura e não invasiva. É uma técnica não traumática, de fácil execução e mais rápida em relação as demais. Porém o espaço retromolar reduzido e grande procedimento utilizando a cavidade oral para acesso pode limitar a utilização dessa técnica ${ }^{12}$.

Por outro lado, a traqueostomia é um método tradicional para controle das vias aéreas em casos do trauma de face severo. Apesar de se rum dos procedimentos cirúrgicos mais comuns no momento do trauma, pode levar a um número elevado de complicações, como hemorragia, lesão ao nervo laríngeo recorrente, pneumonia, pneumotórax, enfisema cirúrgico, obstrução do tubo, estenose traqueal, traqueomalácia, granuloma e cicatriz pobre esteticamente. Onde elas são encontradas com frequência ${ }^{7,8,11,12}$.

\section{CONSIDERAÇÕES FINAIS}

É possível concluir que a intubação submentoniana é uma alternativa segura, eficaz, e com baixo índice de complicações em comparação a traqueostomia e pode ser indicada quando a intubação nasotraqueal e orotraqueal é contraindicada e quando existe necessidade de manutenção da oclusão dentária ou abordagem ao nariz são necessários durante a cirurgia. Embora diversas alterações tenham sido feitas a partir da técnica inicial, essas alterações não se mostraram superiores.

\section{REFERÊNCIAS}

1. Rodrigues WC, Melo WM, Almeida RS, PardoKaba SC, Sonoda CK, Shinohara EH. Submental intubation in cases of panfacial fractures: a retrospective study. Anesth Prog. 2017; 64(3):153-61.

2. Cheong Y, Kang SS, Kim M, Son HJ, Park J, Kim J. Submental intubation in patients with complex maxillofacial injuries. J Lifestyle Med. 2016; 6(2):68-71.

3. Kumar RR, Vylopilli S, Sayd S, Thangavelu A, Joseph B, Ahsan A. Submental intubation: alternative short-term airway management in maxillofacial trauma. J Korean Assoc Oral Maxillofac Surg. 2016;42(3):151-56. 
4. Kaiser A, Semanoff A, Christensen L, Sadoff D, Digiacomo J. Submental intubation: an underutilized technique for airway management in patients with panfacial trauma. J Craniofac Surg. 29(5):1349-51.

5. Akbari H, Heidari-Gorji MA, Poormousa R, Ayyasi M. Submental intubation in maxillofacial fracture: a case report. J Korean Assoc Oral Maxillofac Surg. 2016;42(3):166-68.

6. González-Magaña F, Malagón-Hidalgo HO, García-Cano E, Vilchis-López R, Fentanes-Vera A, Ayala-Ugalde FA. Airway management through submental derivation: a safe and easily reproduced alternative for patients with complex facial trauma. J Korean Assoc Oral Maxillofac Surg. 2018;44(1):12-17.

7. Kita R, Kikuta T, Takahashi M, Ootani T, Takaoka M, Matsuda $M$ et al. Efficacy and complications of submental tracheal intubation compared with tracheostomy in maxillofacial trauma patients. J Oral Sci.2016;58(1):23-8.

8. Lim D, Ma BC, Parumo R, Shanmuhasuntharam P. Thirty years of submental intubation: a review. Int J Oral Maxillofac Surg. 2018;47(9):1161-65.

9. Savitha KS, Kujur AR, Vikram MS, Joseph S. A modified submental orotracheal intubation. Anesth Essays Res. 2016;10(1):132-35.

10.Ujam A, Perry M. Minimally traumatic submental intubation: a novel dilational technique. Eur J Trauma Emerg Surg, 2017;43(3):359-62.

11.Ali S, Athar M, Ahmed SM, Siddiqi OA, Badar A. A randomized control trial of awake oral to submental conversion versus asleep technique in maxillofacial trauma. Ann Maxillofac Surg. 2017;7(2):202-6.

12. Shivpuri A. Sub-mental Intubation in Oral and Maxillofacial Trauma Patients. Indian J Surg. 2015;77(Suppl 3):1450-52.

\section{CONFLITO DE INTERESSES}

Os autores declaram não haver conflitos de interesse.

\section{AUTOR PARA CORRESPONDENCIA}

\section{Gabriel Mulinari dos Santos}

gabriel_mulinari@hotmail.com

Submetido em 12/07/2019

Aceito em 21/09/2019 